\title{
Encouraging replication and reproducible research
}

\author{
Rob J Hyndman
}

30 November 2009

It has become standard in most sciences for results to be replicated before being widely accepted. Remember cold fusion? Research findings that cannot be independently verified under the same or very similar conditions are little more than published opinions. In fact, the painstaking step-by-step duplication of published research is often the only way to properly assess the work done by others (Peng et al., 2006; Laine et al., 2007). While replicating research is accepted practice in medicine, chemistry, physics, and many other areas of science, it has not been part of the research culture in statistics, econometrics and other fields associated with forecasting.

For many years, the International Journal of Forecasting (IJF) has given lip-service to the need for replication, but we have not done much to encourage it. I would like to change this situation. As the leading journal in forecasting, the IJF has a responsibility to set research standards.

So, a couple of years ago, we started asking authors to make their data and code available on our website. Then last year we changed our guidelines for authors to say

Authors will normally be expected to submit a complete set of any data used in electronic form, or provide instructions for how to obtain them. Exceptions to this requirement may be made at the discretion of the handling editor. The author must describe methods and data sufficiently so the research can be replicated. The provision of code as well as data is encouraged, but not required.

This is consistent with the moves of many granting agencies which are now starting to require publicly funded research to make the data publicly available. Once the data are public, other researchers can verify (or otherwise) the conclusions drawn.

Six months ago, the International Journal of Forecasting website (www. forecasters.org/ijf) was redesigned to allow supplements and comments on each published paper. Supplementary information about a paper can be provided by authors and is freely available online. This can 
include data, computer code, large tables, extra figures, extended footnotes, extra relevant material, etc. Authors are required to provide whatever material is needed allow their results to be replicated without excessive difficulty.

There are some recent software tools available to help facilitate reproducibility of research. See Koenker \& Zeilis (2009) for an excellent introduction to these developments.

It is also now possible for readers to contribute comments on each IJF paper via the website. These comments are publicly visible. The authors may provide responses if they choose to do so. So if you have any thoughts on published papers, you can now make them public for the benefit of all forecasters. You can think of this as an extension of refereeing, but post-publication and publicly visible. All comments will be moderated.

These comments are not intended to be extensive, and many papers will likely have no comments. Rather, they are intended to provide a forum for any public post-publication discussion of a paper. Here are some examples of how the commenting system might be used.

1. The findings of an IJF paper are replicated, extended or overturned. Then readers and authors are encouraged to post a comment on the IJF paper, providing a reference to the later work with a brief explanation.

2. An author discovers an error in a published paper. Rather than publish a corrigendum (as we have done in the past), errata will now be made available via online comments.

3. A researcher develops an $\mathrm{R}$ package implementing the method described in an IJF paper. They can use the comments to add a link to their package.

4. A reader disagrees with statements made in an IJF paper. They can post a comment explaining their disagreement. (In this case, the author will be invited to respond.)

Online supplements and comments are available on all papers published in the IJF, back to volume 1 . So if authors of previously published papers have additional information they would like to contribute, please send it to me at ijf@forecasters.org and it will be added to the website. Online supplements and comments are moderated but not refereed.

The journal is also encouraging replication studies by publishing them. Two years ago, we published Gardner \& Diaz-Saiz (2008) which attempted to replicate Fildes et al. (1998) and provided some useful new insight into the original results. We welcome submission of other replication papers, especially when they provide new insights. 
Finally, we invited Heiner Evanschitzky and Scott Armstrong to write a paper on replications in forecasting research. We hope that Evanschitzky \& Armstrong (2010), along with the new facilities on our website, will encourage forecasters to see replication studies as an important part of the research process.

\section{References}

Evanschitzky, H. \& Armstrong, J. S. (2010). Replications of forecasting research. International Journal of Forecasting, 26, this issue.

Fildes, R., Hibon, M., Makridakis, S., \& Meade, N. (1998). Generalising about univariate forecasting methods: Further empirical evidence. International Journal of Forecasting, 14, 339-358.

Gardner, Jr., E. S. \& Diaz-Saiz, J. (2008). Exponential smoothing in the telecommunications data. International Journal of Forecasting, 24, 170-174.

Koenker, R. \& Zeilis, A. (2009). On reproducible econometric research. Journal of Applied Econometrics, 24(5), 833-847.

Laine, C., Goodman, S. N., Griswold, M. E., \& Sox, H. C. (2007). Reproducible research: moving toward research the public can really trust. Annals of Internal Medicine, 146(6), 450-453.

Peng, R. D., Dominici, F., \& Zeger, S. L. (2006). Reproducible epidemiologic research. American Journal of Epidemiology, 163(9), 783-789.

Rob J Hyndman

Editor-in-Chief, International Journal of Forecasting

Monash University

Clayton VIC 3800, Australia

ijf@forecasters.org 